\title{
A phase-space study of the quantum Loschmidt Echo in the semiclassical limit
}

\author{
Monique Combescure \\ IPNL, Bâtiment Paul Dirac \\ 4 rue Enrico Fermi, Université Lyon-1 \\ F.69622 VILLEURBANNE Cedex, France \\ email : monique.combescure@ipnl.in2p3.fr \\ Didier Robert \\ Département de Mathématiques \\ Laboratoire Jean Leray, CNRS-UMR 6629 \\ Université de Nantes, 2 rue de la Houssinière, \\ F-44322 NANTES Cedex 03, France \\ email : Didier.Robert@math.univ-nantes.fr
}

\begin{abstract}
The notion of Loschmidt echo (also called "quantum fidelity") has been introduced in order to study the (in)-stability of the quantum dynamics under perturbations of the Hamiltonian. It has been extensively studied in the past few years in the physics literature, in connection with the problems of "quantum chaos", quantum computation and decoherence.

In this paper, we study this quantity semiclassically (as $\hbar \rightarrow 0$ ), taking as reference quantum states the usual coherent states. The latter are known to be well adapted to a semiclassical analysis, in particular with respect to semiclassical estimates of their time evolution. For times not larger than the so-called "Ehrenfest time" $C|\log \hbar|$, we are able to estimate semiclassically the Loschmidt Echo as a function of $t$ (time), $\hbar$ (Planck constant), and $\delta$ (the size of the perturbation). The way two classical trajectories merging from the same point in classical phase-space, fly apart or come close together along the evolutions governed by the perturbed and unperturbed Hamiltonians play a major role in this estimate.

We also give estimates of the "return probability" (again on reference states being the coherent states) by the same method, as a function of $t$ and $\hbar$.
\end{abstract}




\section{Introduction}

The semiclassical time behaviour of quantum wavepackets has been the subject of intense interest in the last decades, in particular in situations where there is some hyperbolicity in the corresponding classical dynamics (Lyapunov exponents) [9], [17], [30]. Moreover the response of a quantum system to an external perturbation when the size $\delta$ of the perturbation increases can manifest intriguing properties such as recurrences or decay in time of the so-called Loschmidt Echo (or "quantum fidelity") [7], [8]. By Loschmidt Echo we mean the following:

starting from a quantum Hamiltonian $\hat{H}$ in $L^{2}\left(\mathbb{R}^{d}\right)$, whose classical counterpart $H$ has a chaotic dynamics, and adding to it a " perturbation" $\hat{H}_{\delta}=\hat{H}+\delta \hat{V}$, then we compare the evolutions in time $U(t):=e^{-i t \hat{H} / \hbar} \quad, \quad U_{\delta}(t):=e^{-i t \hat{H}_{\delta} / \hbar} \quad$ of initial quantum wavepackets $\varphi$ sufficiently well localized around some point $z$ in phasespace; more precisely the overlap between the two evolutions, or rather its square absolute value, is:

$$
F_{\hbar, \delta}(t):=\left|\left\langle U_{\delta}(t) \varphi, U(t) \varphi\right\rangle\right|^{2}
$$

For example for quantum dynamics in Hilbert space $\mathcal{H}=L^{2}\left(\mathbb{R}^{d}\right), d$ being the space dimension, $\varphi$ can be chosen as the usual coherent states, since they are the quantum wavepackets "as most localized as possible" in phase-space $\mathbb{R}^{2 d}$.

Since for $\delta=0$, we obviously have $F_{\hbar, 0}(t) \equiv 1$, and for any $\delta, F_{\hbar, \delta}(0)=1$, the type of decay in $t$ of $F_{\hbar, \delta}(t)$ so to say measures the (in)fidelity of the quantum evolution with respect to a perturbation of size $\delta$ for generic initial wavepackets $\varphi$.

The notion of Loschmidt Echo seems to have been first introduced by Peres ([24]), in the following spirit: since the sensitivity to initial data which characterizes classical chaos has no quantum counterpart because of unitarity of the quantum evolution, at least the "sensitivity to perturbations" of the Hamiltonian could replace it as a characterization of chaoticity in the "quantum world".

A big amount of recent work appeared on the subject, studying in an essentially heuristic way the decay in time of $F_{\hbar, \delta}(t)$ as $t$ increases from zero to infinity; some of them also study this point in relationship with the important question of decoherence. (See [1], 5], [12-14], [18], [22-26], [30-32]). 
In this "jungle" of sometimes contradictory results, it is hard to see the various arguments involved, in particular the precise behaviour of $F_{\hbar, \delta}(t)$ as $\delta$ (the size of the perturbation), $t$ (the time), and of course $\hbar$ (the Planck constant) are varied, in particular in which sense and order the various limts $\delta \rightarrow 0, \hbar \rightarrow 0, t \rightarrow \infty$ are taken.

Also an important point to consider is how $F_{\hbar, \delta}(t)$ depends on the location of the phase-space point $z$ around which the initial wavepacket $\varphi$ is peaked (since classical chaoticity distinguishes various zones in phase-space with "more or less regularity properties").

The aim of the present paper is to start a rigorous approach of the question of semiclassical estimate of $F_{\hbar, \delta}(t)$, in terms of classical characteristics of the (perturbed and unperturbed classical flows), for initial wavepackets $\varphi=\varphi_{z}$ being the coherent state at phase-space point $z$. These estimates are non-perturbative, and are carefully calculated in terms of parameters $(z, \delta, t, \hbar)$. The main tools we have used and developed in this respect are

1) semiclassical coherent states propagation estimates ([9])

2) a beautiful formula inspired by B. Mehlig and M. Wilkinson ([22]) about the Weyl symbol of a metaplectic operator, and thus of its expectation value in coherent states as a simple Gaussian phase-space integral ( see [10] where we have completed the proof of Mehlig-Wilkinson, and treated in particular the case where the monodromy operator has eigenvalue 1).

Note that very recently, J. Bolte and T. Schwaibold have independently obtained a similar result about semiclassical estimates of the Quantum Fidelity ([2]).

The plan of this paper is as follows. In section 2 we give some preliminaries about the Echo for suitable quantum observables, and give the semiclassics of it. In Section 3 , we consider the (integrable) $d=1$ case, and consider the "return probability" in the semiclassical limit. We give a mathematical rigorous presentation of beautiful results on "quantum revivals" obtained by physicists twenty years ago (see [23, [32, [20]). In Section 4 we consider the general $d$-dimensional case and give a semiclassical calculus of the "return probability" and of the quantum fidelity, with precise error estimates. 


\section{Preliminaries}

Let us consider the quantum Hamiltonian $\hat{H}_{\delta}=\hat{H}_{0}+\delta \hat{V}$, depending on a real parameter $\delta . \hat{H}_{\delta}$ is the Weyl quantization of smooth classical observables defined on the phase space $\mathbb{R}^{2 d}$. Our assumptions on $H_{\delta}$ are as follows:

Assumptions :

(H1) $H_{\delta} \in C^{\infty}\left(\mathbb{R}^{2 d}\right)$ and $\left|\partial_{X}^{\gamma} H_{\delta}(X)\right| \leq C_{\gamma}, \forall X \in \mathbb{R}^{2 d}, \forall \gamma:|\gamma| \geq 2$

or

(H'1) There exist a bounded open set $\Omega \subseteq \mathbb{R}^{2 d}$ such that $\Omega$ is left invariant by the classical flow $\phi_{\delta}^{t}, \forall t, \delta \in \mathbb{R}$ defined by the classical Hamiltonian $H_{\delta}$. We assume that the $\hbar$-Weyl quantization $\hat{H}_{\delta} \equiv \mathrm{Op}_{\hbar}^{w} H_{\delta}$ of $H_{\delta}$ is a self-adjoint operator in $L^{2}\left(\mathbb{R}^{d}\right)$ for all $\delta \in \mathbb{R}$.

Let $L \in \mathcal{C}^{\infty}\left(\mathbb{R}^{2 d}\right)$ be a classical observable and $\hat{L}=\mathrm{Op}_{\hbar}^{w} L$. Then we assume:

(H2) $L \in \mathcal{S}\left(\mathbb{R}^{2 d}\right)$ if (H1) is satisfied,

(H'2) $L \in \mathcal{C}_{0}^{\infty}(\Omega)$ if (H'1) is satisfied, where $C_{0}^{\infty}(\Omega)$ is the linear space of $C^{\infty}$-smooth functions with compact support in $\Omega$.

Let us consider the time evolution unitary operator $U_{\delta}(t)$, in the Hilbert space $\mathcal{H}=L^{2}\left(\mathbb{R}^{d}\right)$,

$$
U_{\delta}(t)=\exp \left(-\frac{i t}{\hbar} \hat{H}_{\delta}\right)
$$

Definition 2.1 (i) The quantum echo is the unitary operator defined by

$$
E_{\delta}^{(q)}(t)=U_{0}(-t) U_{\delta}(t)
$$

(ii) The quantum fidelity, for a state $\psi_{0},\left\|\psi_{0}\right\|=1$, is defined by

$$
f_{\delta}^{(q)}(t)=\left|\left\langle\psi_{0}, E_{\delta}^{(q)}(t) \psi_{0}\right\rangle\right|^{2}
$$

(iii) Let $L_{\delta}(t)$ be the following time-dependent quantum observable:

$$
\hat{L}_{\delta}(t):=E_{\delta}^{q}(t)^{-1} \hat{L} E_{\delta}^{q}(t)
$$

The notion of "fidelity" was introduced first in classical mechanics by Loschmidt (in discussions with Boltzmann) then adapted in quantum mechanics by Peres [24]. 
Let us define $\phi_{\delta}^{t}$, the classical flow defined in the phase space $\mathcal{Z} \equiv \mathbb{R}^{2 d}$ by the classical Hamiltonian $H_{\delta}$. Recall that $z_{\delta, t}:=\phi_{\delta}^{t}\left(z_{0}\right)$ is the solution of the differential equation $\dot{z}_{t}=J \nabla H_{\delta}\left(z_{t}\right), z_{t=0}=z_{0}$. So that the "classical echo" is defined by

$$
E_{\delta}^{(c l)}(t, X):=\phi_{0}^{-t} \circ \phi_{\delta}^{t}(X) .
$$

Here $J$ is the symplectic matrix given as:

$$
J:=\left(\begin{array}{cc}
0 & \mathbb{1}_{d} \\
-\mathbb{1}_{d} & 0
\end{array}\right)
$$

and $\mathbb{1}_{d}$ is the identity $d \times d$ matrix.

We can see easily that in the semiclassical limit, $\hbar \rightarrow 0$, the quantum echo converges to the classical echo. In more mathematical terms, the quantum echo is a $\hbar$ Fourier Integral Operator whose canonical relation is the classical echo. This is a consequence of the semiclassical Egorov theorem as we shall see now, at least when the reference quantum state is a "coherent state". Let us recall here the definition of a (Gaussian) coherent state which will be used later:

Given $\varphi_{0}(x):=(\pi \hbar)^{-d / 4} \exp \left(-x^{2} / 2 \hbar\right)$, we define, for $z:=(q, p) \in \mathbb{R}^{2 d}$ :

$$
\varphi_{z}:=\hat{T}(z) \varphi_{0}, \text { where } \hat{T}(z):=\exp \left(\frac{i(p \cdot \hat{Q}-q \cdot \hat{P})}{\hbar}\right)
$$

are the translation Weyl operators.

Proposition 2.2 Assume either (H1-H2) or (H'1-H'2) for the Hamiltonians $H_{\delta}$ and observables $L$.

(i) We have

$$
\lim _{\hbar \rightarrow 0}(2 \pi \hbar)^{d} \operatorname{Tr}\left(\hat{L} \hat{L}_{\delta}(t)\right)=\int_{\mathbb{R}^{2 d}} L(X) L\left(E_{\delta}^{(c l)}(t, X)\right) d X
$$

(ii) Let be $\varphi_{z}$ the coherent state living at $z$. Then we have:

$$
\lim _{\hbar \rightarrow 0}\left\langle E_{\delta}^{(q)}(t) \varphi_{z} \mid \hat{L} E_{\delta}^{(q)}(t) \varphi_{z}\right\rangle=L\left(E_{\delta}^{(c l)}(t, z)\right)
$$

Moreover there exists $C>0$ such that the limits are uniform as long as $|t| \leq$ $C|\log \hbar|$. If $H_{0}$ is integrable in $\Omega$ and if $\delta=\mathcal{O}(\hbar)$, then the limit is uniform as long as $|t| \leq C_{\varepsilon} \hbar^{-1 / 3+\varepsilon}$

Proof: It follows from the semiclassical Egorov Theorem,with improvement for large times derived by Bouzouina-Robert ([3] ). 
Remark 2.3 An important question is to control the time of validity of the semiclassical approximation. Rigorous mathematical results are far from numerical and theoretical expected physical results. Without assumptions on classical flows this time is the Ehrenfest time (of order $\log \left(\hbar^{-1}\right)$ ).

\section{Revivals for 1-D systems}

In this Section we shall consider the Return Probability, which is a simplified form of Quantum Fidelity as we shall explain in the next Section. For one-dimensional problems, this Return Probability manifests interesting recurrences very close to 1 , as time evolves. This phenomenon was studied in the physics literature to understand time evolution of Rydberg atoms and their quantum beats, with decay and reformation of the wave packet (see for example [23, 20], [32] and references herein contained). In this section we want to give a flavour of results obtained by physicists in the last twenty years, concerning revivals for the quantum return probability ([32] for a very clear and detailed review) and show how to put them in a more rigorous mathematical framework. Let us consider a classical 1-D Hamiltonian $H$. One assumes $H$ to be a smooth, confining with one well Hamiltonian. This means that the energy surface $H^{-1}(E)$ has only one connected component in phase-space $\mathcal{Z}$. Let $\Psi_{n}$ be an orthonormal basis of eigenstates, with eigenvalues $E_{n}, n \in \mathbb{N}$.

Let $\psi_{0}=\sum_{n \in \mathbb{N}} c_{n} \Psi_{n}$ an initial normalized state, and $\psi_{t}=U(t) \psi_{0}$. Then the autocorrelation fonction is :

$$
a(t):=\left\langle\psi_{0} \mid \psi_{t}\right\rangle=\sum_{n \in \mathbb{N}}\left|c_{n}\right|^{2} \mathrm{e}^{-\frac{i t}{\hbar} E_{n}}
$$

and the return probability is defined by

$$
\rho(t)=|a(t)|^{2}
$$

Let us remark here that $a$ is an almost periodic function (in the sense of H. Bohr) in time $t$ on $\mathbb{R}$. Therefore, for every $\varepsilon>0$, there exists $T_{\varepsilon}>0$ and for every $k \in \mathbb{Z}$ there exists $t_{k} \in\left[k T_{\varepsilon},(k+1) T_{\varepsilon}\right.$ [ such that $\left|a\left(t_{k}\right)-1\right| \leq \varepsilon$. This fact can be interpreted as a quantum analog of the famous return Poincaré in classical mechanics.

But we have no information here on the almost return time $t_{k}$, in particular when $\hbar$ tends to zero. For 1-D systems much more accurate results are available because for these systems the spectrum can be computed with error $\mathcal{O}\left(\hbar^{\infty}\right)$ according the BohrSommerfeld quantization rule. Recall here this result. We take the presentation 
from the paper by Helffer-Robert $([18])$ and we refer to this paper for more details. (see also the thesis of Bily for a proof using coherent states).

Let us give now the sufficient assumptions:

$\left(A_{1}\right) H(z)$ is real valued, $H \in C^{\infty}\left(\mathbb{R}^{2}\right)$.

$\left(A_{2}\right) H$ is bounded below 1 : there exist $c_{0}>0$ and $\gamma_{0} \in \mathbb{R}$ such that $c_{0} \leq H(z)+\gamma_{0}$. Furthermore $H(z)+\gamma_{0}$ is supposed to be a temperate weight, i.e there exist $C>$ $0, M \in \mathbb{R}$, such that:

$$
H(z)+\lambda_{0} \leq C\left(H\left(z^{\prime}\right)+\lambda_{0}\right)\left(1+\left|z-z^{\prime}\right|\right)^{M} \quad \forall z, z^{\prime} \in Z .
$$

$\left(A_{3}\right) \forall \gamma$ multiindex $\exists c>0$ such that: $\left|\partial_{z}^{\gamma} H\right| \leq c\left(H+\lambda_{0}\right)$.

We want to consider here bound states of $\hat{H}$ in a fixed energy band. So, let us consider a classical energy interval $I=] E_{-}-\varepsilon, E_{+}+\varepsilon\left[, \quad E_{-}<E_{+}\right.$such that we have:

$\left(A_{4}\right) H^{-1}(I)$ is a bounded set of the phase space $\mathbb{R}^{2}$.

This implies that in the closed interval $I=\left[E_{-}, E_{+}\right]$, for $\hbar>0$ small enough, the spectrum of $\hat{H}$ in $I$ is purely discrete $([18])$.

For some energy level $E \in I$, let us introduce the assumption :

$\left(A_{5}\right) E$ is a regular value of $H$. That means: $H(x, \xi)=E \Rightarrow \nabla_{(x, \xi)} H(x, \xi) \neq 0$.

Furthermore we assume that for every $E \in I, H^{-1}(E)$ is a connected curve.

Let us consider a non critical energy interval $\left[E^{-}, E^{+}\right]$. It is well konwn that the action integral is $\mathcal{J}(E)=\int_{H(z) \leq E} d z$ and the period along the energy curve $H^{-1}(E)$ is $T_{E}=\mathcal{J}^{\prime}(E), E \in\left[E^{-}, E^{+}\right]$. Let us denote $F^{ \pm}=\mathcal{J}\left(E^{ \pm}\right)$. The eigenvalues of $\hat{H}$ in $\left[E^{-}, E^{+}\right]$are determined by the following Bohr-Sommerfeld rule.

Theorem 3.1 ([18]) Under the assumptions $\left(A_{1}\right)$ to $\left(A_{5}\right)$, there exists a $C^{\infty}$ function on $\left[F^{-}, F^{+}\right], F \mapsto b(F, \hbar)$ and $C^{\infty}$ functions $b_{j}$ defined on $\left[F^{-}, F^{+}\right]$such that $b(F, \hbar)=\sum_{j \in \mathbb{N}} b_{j}(F) \hbar^{j}+\mathcal{O}\left(\hbar^{\infty}\right)$ and the eigenvalues $E_{n}$ of $\hat{H}$ in $I$ are given by

$$
\begin{array}{r}
E_{n}=b\left(\left(n+\frac{1}{2}\right) \hbar, \hbar\right)+\mathcal{O}\left(\hbar^{\infty}\right), \text { for } n \text { such that }\left(n+\frac{1}{2}\right) \hbar \in\left[F^{-}, F^{+}\right] \\
\text {where } b_{0}(F)=2 \pi \mathcal{J}^{-1}(F) \text {, and } b_{1}=0 .
\end{array}
$$

Remark 3.2 In recent papers [6], [21] the authors have given some methods to compute explicitly the terms $b_{j}$ for $j \geq 2$ in the expansion in $\hbar$ in the Bohr-Sommerfeld rule.

\footnotetext{
${ }^{1}$ Using the semi-classical functional calculus 29 it is not a serious restriction
} 
Let us now choose an initial wave packet, $\psi=\sum_{n} c_{n} \Psi_{n}$, tightly spread around the energy $E_{\bar{n}}$ where $E_{\bar{n}}=b\left(\bar{n}+\frac{1}{2}, \hbar\right)$ and $\bar{n}$ is a family of given quantum numbers, depending on $\hbar$, and such that $\left(\bar{n}+\frac{1}{2}\right) \hbar \in\left[F^{-}, F^{+}\right]$for every $\left.\left.\hbar \in\right] 0, \hbar_{0}\right]$. Let us choose the coefficient $c_{n}$ defined by

$$
c_{n}=K_{\tau, \hbar} \chi_{1}\left(\frac{E_{n}-E_{\bar{n}}}{\tau_{\hbar}}\right) \chi_{0}\left(\frac{E_{n}-E}{\epsilon_{\hbar}}\right)
$$

where $\chi_{1} \in \mathcal{S}(\mathbb{R}), \chi_{0}$ has a bounded support, $\left.\operatorname{supp}\left[\chi_{0}\right] \subseteq\right]-1,1\left[, \chi_{0}(x)=1\right.$ on $[-1 / 2,1 / 2]$, and $K_{\tau, \hbar}$ is defined such that the $L^{2}$-norm of the wave packet is $\sum_{n \in \mathbb{N}}\left|c_{n}\right|^{2}=1$. We shall choose $\tau_{\hbar}$ and $\epsilon_{\hbar}$ such that $\frac{\tau_{\hbar}}{\epsilon_{\hbar}}=\mathcal{O}\left(\hbar^{\delta}\right)$, for some $\delta>0$.

Remark 3.3 From a physical point of view, a state $\psi$ as above is prepared by exciting an atom with a laser beam. The new object is a Rydberg atom.

Pratically, we shall choose $\tau_{\hbar}=\hbar^{\theta}$. We define: $\sigma \equiv \frac{\tau}{\hbar}=\hbar^{\theta-1}$ and $\epsilon_{\hbar}=\hbar^{\theta^{\prime}}$, with $0<\theta^{\prime}<\theta<1$. It is more suggestive for us to keep the notations $\tau_{\hbar} \sigma_{b}$ ar and $\epsilon_{\hbar}$. Let us apply the Taylor formula to $b(F, \hbar)$ around $\bar{F}=\left(\bar{n}+\frac{1}{2}\right) \hbar$.

$$
E_{n}-E_{\bar{n}}=\hbar b_{0}^{\prime}(n-\bar{n})+\frac{\hbar^{2}}{2} b_{0}^{\prime \prime}(n-\bar{n})^{2}+\frac{\hbar^{3}}{6} b_{0}^{\prime \prime \prime}(n-\bar{n})^{3}+\hbar^{3} b_{2}^{\prime}(n-\bar{n})+\mathcal{O}\left(\tau^{4}\right),
$$

if $|n-\bar{n}| \leq C \sigma$, with $C>0$ and where the derivatives of $b_{j}$ in $F$ are computed in $\bar{F}$. Up to a small error in $\hbar$, it is possible to change the definitions of $\chi_{1}$ and $K_{\tau, \hbar}$ such that $c_{n}=K_{\tau, \hbar} \chi\left(\frac{n-\bar{n}}{\sigma}\right)$, with $\sigma=\frac{\tau}{\hbar}$. Let us remark that $K_{\tau, \hbar}$ is of order $\sigma_{\hbar}^{-1}$. This is easily seen from the following lemma.

Lemma 3.4 With the previous notations and assumptions we have

$$
\lim _{\hbar \rightarrow 0}\left(\frac{\hbar}{\tau} \sum_{n}\left|\chi_{1}\left(\frac{E_{n}-E_{\bar{n}}}{\tau_{\hbar}}\right) \chi_{0}\left(\frac{E_{n}-E}{\epsilon_{\hbar}}\right)\right|^{2}\right)=\int_{\mathbb{R}}\left|\chi_{1}(x)\right|^{2} d x
$$

Proof. Besides the assumptions, we make use of formula (3.5) and of the following well known estimate for the number of bound states

$$
\#\left\{n, E_{n} \in[E-\epsilon, E+\epsilon]\right\}=\mathcal{O}\left(\frac{\epsilon}{\hbar}\right) .
$$

The details are left to the reader.

Let us denote by $a_{i}(t)$ the approximation for $a(t)$ obtained by plugging in (3.1) the i-first terms of the Taylor expansion (3.5) denoted by $\kappa_{i}(n)(1 \leq i \leq 3)$. So we get the following preliminary result: 
Proposition 3.5 We have

$$
|a(t)|^{2}=\left|a_{i}(t)\right|^{2}+\mathcal{O}\left(|t| \hbar^{-1} \tau^{i+1}\right)
$$

In particular, $\left|a_{i}(t)\right|^{2}$ is a semiclassical approximation for $|a(t)|^{2}$ valid for times $t$ such that $|t|$ is less than $\hbar^{1+\varepsilon} \tau^{-1-i}$ for any $\varepsilon>0$, with a reminder term $\mathcal{O}\left(\hbar^{\varepsilon}\right)$.

Corollary 3.6 For every $\varepsilon>0$, we can choose $\theta<1$, close enough to 1 , such that

$$
|a(t)|^{2}=\left|a_{i}(t)\right|^{2}+\mathcal{O}\left(|t| \hbar^{i-\varepsilon}\right)
$$

From the proposition and its corollary we can give a mathematical proof for the collapses and revivals phenomenon concerning the return probability $\rho(t)$.

Let us remark first that $\kappa_{1}(n)=b_{0}^{\prime}(n-\bar{n})$ so $\left|a_{1}(t)\right|^{2}$ is periodic with period $T_{c l}=\frac{2 \pi}{b_{0}^{\prime}}$ (classical period along the orbit of energy $\bar{E}$ ). So the return probability $\rho(t)$ is close to 1 for $t=N T_{c l}$ as far as $|t|$ is less than $\tau^{-2} \hbar^{1+\varepsilon}=\mathcal{O}\left(\hbar^{-1+\varepsilon^{\prime}}\right)\left(\varepsilon^{\prime}>\varepsilon\right.$. $)$

For larger times, we have to consider $\kappa_{2}(n)=b_{0}^{\prime}(n-\bar{n})+\frac{\hbar}{2} b_{0}^{\prime \prime}(n-\bar{n})^{2}$ and a second time scale dependent on $\hbar$, the revival time, defined as $T_{r e v}=\frac{4 \pi}{\hbar b_{0}^{\prime \prime}}$. Let us introduce the integer $N=\left[\frac{T_{R e v}}{T_{c} \ell}\right]$ and take $N T_{c \ell}$ as a new time origin. If $t=N T_{c \ell}+s$ we have

$$
a_{2}(t)=\sum\left|c_{m+\bar{n}}\right|^{2} \exp \left(\frac{2 i \pi}{T_{c \ell}} s m\right) \exp \left(\frac{2 i \pi}{T_{R e v}}\left(s-\theta T_{c \ell}\right) m\right),
$$

where $\theta \in[0,1[$. Therefore we have

$$
a\left(N T_{c \ell}+s\right)=a(s)+\mathcal{O}\left(\hbar^{\varepsilon}\right), \text { as long as }|s| \leq \hbar^{\varepsilon-2}
$$

So, around the time $N T_{c \ell}$, which is of order $\hbar^{-1}$, the signal retains its initial form and moves according to the classical laws.

Now we shall prove that for large time intervals, below the time $T_{\text {Rev }}$ the signal $a(t)$ is very small. Let us consider

$$
J_{\hbar}=\left[\hbar^{1-2 \theta-\delta_{1}}, \hbar^{\delta_{2} / 2-\theta}\right]
$$

where $\delta_{1}>0, \delta_{2}>0$ are any small fixed real numbers satisfying $\delta_{2}+\delta_{1} / 2+\theta<1$.

Proposition 3.7 Under the previous assumptions and notations, we have

$$
\lim _{\hbar \rightarrow 0, t \in J_{\hbar}} \rho(t)=0
$$


Proof: For simplicity, we shall prove the collapse property (3.10) for a Gaussian cut-off, $\chi_{1}(x)=\mathrm{e}^{-x^{2} / 4}$. We can assume that $T_{c \ell}=2 \pi$.

The trick here is to apply the Poisson formula in the time variable to

$$
a_{2}(t)=K_{\tau, \hbar} \sum_{m \in \mathbb{Z}} \exp \left(-\frac{m^{2}}{2 \sigma^{2}}+2 i \pi t \frac{m^{2}}{T_{\text {rev }}}\right) \exp (i t m)
$$

So, applying the classical formula for the Fourier transform of a Gaussian we get

$$
a_{2}(t)=K_{\tau, \hbar} \sqrt{\frac{2 \pi}{\gamma_{t, \hbar}}} \sum_{\ell \in \mathbb{Z}} \exp \left(-2 \pi^{2} \frac{\left(\ell-\frac{2 \pi t}{2 \pi}\right)^{2}}{\gamma_{t, \hbar}}\right)
$$

where $\gamma_{t, \hbar}=\left(\frac{1}{\sigma^{2}}-\frac{4 i \pi t}{T_{r e v}}\right)$.

We have

$$
\gamma_{t, \hbar}=\gamma_{0, \hbar}\left(1-\frac{4 i \pi t}{T_{\text {rev }}} \sigma^{2}\right)
$$

and each Gaussian term in the sum in (3.12) has width $\delta_{t}$, given by

$$
\left.\delta_{t}=\left(\Re\left(\gamma_{t, \hbar}{ }^{-1}\right)\right)\right)^{-1 / 2}=\left(\frac{1}{\sigma^{2}}+16 \pi^{2} \frac{t^{2} \sigma^{2}}{T_{r e v}{ }^{2}}\right)^{1 / 2}
$$

From formula (3.12), we can see that a sufficient condition for $t$ to be a collapse time for $\rho(t)$ is that $\left|\frac{\gamma_{0, \hbar}}{\gamma_{t, \hbar}}\right|$ and $\delta_{t}$ tend to 0 with $\hbar$. Therefore we get easily (3.10).

Remark 3.8 The length of $J_{\hbar}$ is of order is of order $\hbar^{\delta_{2} / 2-\theta}$. So the length of $J_{\hbar}$ is very large for $\hbar$ very small (remember that $\delta_{2}$ is small and $\theta$ close to 1 ). Therefore in the large intervall $J_{\hbar}, a(t)$ is very small and in particular its classical period $T_{c e}$ has disappeared. But we have seen that this period appears again at time $N T_{c \ell}$ (close to $T_{\text {Rev }}$, for small $\hbar$. These facts justify the name "revival" given to this phenomenon. As it is shown in [32], it is also possible to observe fractional revivals, using some elementary properties of integers.

Remark 3.9 The above analysis could be extended to completely integrable systems in d degree of freedom, using the corresponding Bohr-Sommerfeld rules [4].

Remark 3.10 In the next section, for d-multidimensional sytems, we shall start with a Gaussian coherent $\varphi_{z}$ of classical energy $E=H(z)$. Let us consider $\chi$ as above and such that $\chi=1$ in a small neighborhood of $E$. Then, modulo an error term $\mathcal{O}\left(\hbar^{\infty}\right)$, we have easily

$$
\left\langle\varphi_{z} \mid U(t) \varphi_{z}\right\rangle=\sum_{n \in \mathbb{N}} \chi\left(\frac{E_{n}-E}{\tau}\right)\left|\left\langle\varphi_{z} \mid e_{n}\right\rangle\right|^{2} \mathrm{e}^{-\frac{i t}{\hbar} E_{n}}
$$


We get something similar to the definition of $a(t)$ but with coefficients $c_{n}$ not necessary smooth in the variable $n$, so application of the Poisson formula seems difficult.

\section{$4 \quad$ Fidelity on coherent states}

Let us recall that the Return Probability for suitable time-dependent Hamiltonians $\hat{H}(t)$ (for which the quantum unitary evolution $U(t, s)$ can be shown to exist) in some quantum state $\psi_{0} \in \mathcal{H}=L^{2}\left(\mathbb{R}^{d}\right)$ is defined as

$$
R(t):=\left|\left\langle\psi_{0}, U(t, 0) \psi_{0}\right\rangle\right|^{2}
$$

It measures the quantum probability that the time-evolved quantum state $U(t, 0) \psi_{0}$ returns close to its initial quantum configuration $\psi_{0}$.

The Quantum Fidelity (2.2) can be related to the Return Probability for a suitable time-dependent Hamiltonian:

$$
\hat{H}(t)=\exp \left(\frac{i t \hat{H}_{0}}{\hbar}\right)\left(\hat{H}_{\delta}-\hat{H}_{0}\right) \exp \left(-\frac{i t \hat{H}_{0}}{\hbar}\right)
$$

Namely, according to Schrödinger equation, we have that, defining $U(t):=U_{0}(-t) U_{\delta}(t)$,

$$
i \hbar \frac{d}{d t} U(t)=\hat{H}(t) U(t)
$$

so that $U(t, s)=U(t) U(-s)$ is the time evolution associated to (4.2).

Thus as a training for studying Quantum Fidelities, let us first consider the semiclassical study of the Return Probability in the coherent states.

Let us assume the following hypotheses:

1. $H(t, X)$ is a smooth time dependent Hamiltonian, continuous in time $t \in \mathbb{R}$, $C^{\infty}$ in $X \in \mathbb{R}^{2 d}$ such that for every multiindex $\alpha$ there exist $C_{\alpha}>0$ and $M_{\alpha} \in \mathbb{R}$ such that

$$
\left|\partial_{X}^{\alpha} H(t, X)\right| \leq C_{\alpha}(1+|X|)^{M_{\alpha}}, \text { for } X \in \mathbb{R}^{2 d}, t \in \mathbb{R}
$$

2. The classical flow $\phi^{t, s}$ generated by $H(t)$ (with initial data at $s$ ) exists for all times $t, s$. We shall denote $\phi^{t}=\phi^{t, 0}$. 
3. $\hat{H}(t):=\mathrm{Op}_{\hbar}^{w} H(t)$ is self-adjoint on $L^{2}\left(\mathbb{R}^{d}\right)$, and generates a strongly continuous evolution operator $U(t, s)$ satisfying the chain rule $U(t, \tau) U(\tau, s)=$ $U(t, s), \forall s, \tau, t \in \mathbb{R}$.

Note that sufficient conditions for this to hold are given in [9].

Then we define the Stability Matrix $F(t)$ for the flow $\phi^{t}$ as follows:

It is the $2 d \times 2 d$ symplectic matrix solution of the following linear problem:

$$
\dot{F}_{t}=J H^{\prime \prime}(t) F_{t}
$$

where $H^{\prime \prime}(t)$ is the Hessian of $H(t)$ taken at point $\phi^{t} z$ of the phase-space trajectory, starting with initial phase-space point $z \in \mathbb{R}^{2 d}, J$ being the symplectic matrix given by (2.3). We have:

Theorem 4.1 Let us assume Hypotheses 1,2,3 above. Then we have, for the amplitude of the return probability $r(t, z):=\left|\left\langle U(t, 0) \varphi_{z}, \varphi_{z}\right\rangle\right|$, the asymptotic formula as $\hbar \rightarrow 0$,

$$
r(t, z)=\left|\operatorname{det}\left(V_{t}\right)\right|^{-1 / 2} \mathrm{e}^{\frac{\Re \triangle_{t}}{\hbar}}+\mathcal{O}(\sqrt{\hbar})
$$

where

$$
V_{t}=\frac{1}{2}\left(\mathbb{1}+F_{t}+i J\left(\mathbb{1}-F_{t}\right)\right)
$$

$F_{t}$ being the stability matrix for the flow, and

$$
\triangle_{t}=\frac{1}{4} \Gamma_{F_{t}}\left(z_{t}-z\right) \cdot\left(z_{t}-z\right)
$$

with $\Gamma_{F_{t}}=(\mathbb{1}+i J)\left(\mathbb{1}+F_{t}\right)\left(2 V_{t}\right)^{-1}(\mathbb{1}-i J)-\mathbb{1}$

In particular if $z$ lies on a periodic orbit $\gamma$ of the classical flow, with period $T_{\gamma}$, and if $F\left(T_{\gamma}\right)$ is unitary, we get:

$$
r\left(T_{\gamma}, z\right)=1+\mathcal{O}\left(\hbar^{1 / 2}\right)
$$

namely we have almost "quantum revival" when $\hbar \rightarrow 0$.

The proof will be very similar to the one we establish below for the Quantum Fidelity.

Let us now consider the fidelity problem. We want to analyze

$$
f_{z}^{\delta}(t):=\left|\left\langle U_{0}(t) \varphi_{z}, U_{\delta}(t) \varphi_{z}\right\rangle\right|^{2}
$$


For the generators of the (time independent) Hamiltonians $\hat{H}_{\delta}$, we assume:

(H0) $\hat{H}_{\delta}=\mathrm{Op}_{\hbar}^{w} H_{\delta}$

(H2) $H_{\delta}$ is a smooth Hamiltonian such that there exists for any multiindex $\gamma$ constants $C_{\gamma}>0$, and $m_{\gamma} \in \mathbb{R}$ such that $\left|\partial_{X}^{\gamma} H_{\delta}(X)\right| \leq C_{\gamma}(1+|X|)^{m_{\gamma}}, \forall X \in \mathbb{R}^{2 d}$.

We denote by $F_{\delta}(t)$ the stability matrix for $H_{\delta}$ (and similarly for $F_{0}(t)$ and $H_{0}$ ), and by $\phi_{\delta}(t)$ the classical flow for $H_{\delta}$, so that the phase-space point of the classical trajectory starting from $z \in \mathbb{R}^{2 d}$ is $z_{t}^{\delta} \equiv \phi_{\delta}^{t} z$.

Then we have:

Theorem 4.2 Assume (HO), (H2).

(i) Then for any $N \geq 1$ we have the asymptotic expansion:

$$
\left\langle U_{0}(t) \varphi_{z}, U_{\delta}(t) \varphi_{z}\right\rangle=\sum_{j=0}^{N} \hbar^{j / 2} e_{j}\left(t, z, \frac{z_{t}^{\delta}-z_{t}^{0}}{\sqrt{\hbar}}\right)+R_{\delta}^{(N)}(t, \hbar)
$$

where

$$
\text { - } e_{j}(t, z, X):=\alpha_{j}(t, z, X) e^{\Lambda_{t, z} X \cdot X}
$$

- $\alpha_{j}(t, z,$.$) is a polynomial of degree \leq 3 j$

- $\Lambda_{t, z}:=\frac{1}{4} \tilde{F}_{0}^{-1} \Gamma_{F} F_{0}^{-1}, \tilde{F}$ being the transpose of $F$

- $\Gamma_{F}:=(\mathbb{1}+i J)(\mathbb{1}+F)(\mathbb{1}+F+i J(\mathbb{1}-F))^{-1}(\mathbb{1}-i J)-\mathbb{1}$

- and $F$ denotes $F:=F_{0}^{-1} F_{\delta}$

$R_{\delta}^{(N)}(t, \hbar)=\mathcal{O}\left(\hbar^{(N+1) / 2}\right)$ is uniform on every interval $[-T, T](0<T<\infty)$.

In particular we have:

$$
e_{0}(t, z) \equiv\left(\operatorname{det}\left(\frac{1}{2}(\mathbb{1}+F+i J(\mathbb{1}-F))\right)\right)^{-1 / 2}
$$

and denoting by $V_{F}$ the following $2 d \times 2 d$ matrix:

$$
\begin{gathered}
V_{F}:=\frac{1}{2}(\mathbb{1}+F+i J(\mathbb{1}-F)) \\
f_{z}^{\delta}(t)=\left|\operatorname{det} V_{F}\right|^{-1} \exp \left(\frac{2}{\hbar} \Re \Lambda_{t, z}\left(z_{t}^{\delta}-z_{t}^{0}\right) \cdot\left(z_{t}^{\delta}-z_{t}^{0}\right)\right)+\mathcal{O}(\sqrt{\hbar})
\end{gathered}
$$


(ii) Moreover, we have, in the sense of quadratic forms the following inequality:

$$
\Re \Lambda_{t, z} \leq-\frac{1}{2+2\|F\|^{2}} \tilde{F}_{0}^{-1} F_{0}^{-1}
$$

where $\|F\|$ is the largest eigenvalue of $F$, and for any symplectic matrix $F$ :

$$
\left|\operatorname{det} V_{F}\right| \geq 1
$$

(iii) $\lim _{\hbar \rightarrow 0} f_{z}^{\delta}(t)=1 \Longleftrightarrow z_{t}^{\delta}=z_{t}^{0}$ and $F \equiv F_{0}^{-1} F_{\delta}$ is a unitary matrix.

Theorem 4.3 Let us assume that $H_{\delta}(t)$ obeys a Gevrey class assumption: $\left(G_{s}\right): \exists \rho>0, \nu \geq 0, C>0, R>0$ such that:

$\forall \gamma \in \mathbb{N}^{2 d},\left|\partial_{X}^{\gamma} H_{\delta}(X)\right| \leq C R^{1+|\gamma|}(\gamma !)^{s} e^{\nu|X|^{1 / s}}, \forall X \in \mathbb{C}^{2 d},|\Im X| \leq \rho, \forall \delta \in\left[0, \delta_{0}\right], \delta_{0}>0$

then with the notations of Theorem 4.2, we have:

(i) $\left|e_{j}(t, z, X)\right| \leq C^{j+1}(j+1)^{\frac{s_{\star}(j+1)}{2}} e^{-\mu|X|}$, where $s_{\star}=2 s-1$.

(ii) $\forall \varepsilon>0 \exists a>0, \exists c>0$ such that for $|X| \geq \varepsilon$ and $j \leq \frac{a}{\hbar^{1 / s_{\star}}}$ we have $\left|e_{j}(t, z, X)\right| \leq$ $\mathrm{e}^{-\frac{c}{\hbar^{1 / s \star}}}$.

(iii) $\exists C>0, c>0$ such that if $N_{\hbar}=\left[\frac{a}{\hbar^{1 / s_{\star}}}\right]$ we have $\forall t:|t| \leq T$ :

$$
\left|\left\langle U_{0}(t) \varphi_{z}, U_{\delta}(t) \varphi_{z}\right\rangle-\sum_{j=0}^{N_{\hbar}} \hbar^{j / 2} e_{j}\left(t, z, \frac{z_{t}^{\delta}-z_{t}^{0}}{\sqrt{\hbar}}\right)\right| \leq C \exp \left(-\frac{c}{\hbar^{1 / s_{\star}}}\right)
$$

The proof of these theorems heavily relies on a result for semiclassical propagation of coherent states (see [9]) which has been revisited in [30], [31]:

Theorem 4.4 Assume (HO), (H2). Then there exists a family of polynomials $\left\{b_{j}(t, x)\right\}_{j \in \mathbb{N}}$ in $d$ real variables $x=\left(x_{1}, \cdots, x_{d}\right)$, with time dependent coefficients, such that for all $\hbar \in] 0,1]$, we have

$$
\left\|U(t) \varphi_{z}-\exp \left(\frac{i \gamma_{t}}{\hbar}\right) \hat{T}\left(z_{t}\right) \Lambda_{\hbar} \widehat{R_{1}}\left(F_{t}\right)\left(\sum_{0 \leq j \leq N} \hbar^{j / 2} b_{j}(t) g\right)\right\|_{L^{2}\left(\mathbb{R}^{d}\right)} \leq C(N, t, \hbar) \hbar^{(N+1) / 2}
$$

such that for every $N \in \mathbb{N}$, and every $T<+\infty$ we have $\sup _{0<\hbar \leq 1,|t| \leq T} C(N, t, z, \hbar)<+\infty$. $g$ is the simple normalized gaussian function $\mathbb{R}^{d} \mapsto \mathbb{R}$ :

$$
g(x):=(\pi)^{-d / 4} \exp \left(-\frac{x^{2}}{2}\right)
$$


Here $\widehat{R}_{1}(F)$ is the usual metaplectic representation (for $\hbar=1$ ) associated to $F$ (see [10]). Moreover $\Lambda_{\hbar}$ is the following unitary transform in $\mathcal{H}$ :

$$
\begin{gathered}
\Lambda_{\hbar} \psi(x)=\hbar^{-d / 4} \psi\left(x \hbar^{-1 / 2}\right) \quad \text { and } \\
\gamma_{t}(z)=\frac{1}{2} \int_{0}^{t} z_{s} \cdot \nabla H\left(z_{s}\right) d s-t H(z)
\end{gathered}
$$

Let us here recall a simple property of the metaplectic representation: if

$$
F=\left(\begin{array}{cc}
A & B \\
C & D
\end{array}\right)
$$

is the $4 d \times d$ block-matrix form of the symplectic matrix $F$, the action of $\widehat{R}_{1}(F)$ on the state $g$ is given by:

$$
\widehat{R}_{1}(F) g=\pi^{-d / 4}(\operatorname{det}(A+i B))^{-1 / 2} \exp \left(\frac{i}{2} \Gamma x \cdot x\right)
$$

with $\Gamma:=(C+i D)(A+i B)^{-1}$

Let us denote by $\psi_{z, t}^{(N)}$ the approximation of $U(t) \varphi_{z}$ given by (4.8).

Let us recall some more accurate estimate obtained in [9] and [30, 31].

(i) Let be $N$ fixed and $R>0$ such that $\left|z_{t}\right| \leq R, \forall t \in \mathbb{R}$. Then there exist $c_{N}>0$, $k_{R}>0$ such that

$$
\hbar^{(N+1) / 2} C(N, t, z, \hbar) \leq c_{N} k_{R}\left(\sqrt{\hbar}\left|F_{t}\right|^{3}\right)^{N+1}(1+|t|)^{N+1}
$$

In particular, in the generic case, we have a positive Lyapunov exponent $\gamma$ such that $\left|F_{t}\right| \leq \mathrm{e}^{\gamma|t|}$, so that the semiclassical approximation is valid for $|t| \leq \frac{1-\varepsilon}{6 \gamma}|\log \hbar|$.

In the integrable case we have $\left|F_{t}\right| \leq c|t|$ and the semiclassical approximation is valid for $|t| \leq \hbar^{-1 / 6+\varepsilon}$, for any $\varepsilon>0$.

(ii) If $H$ satisfies the following analyticity assumption in the set

$$
\Omega_{\rho}=\left\{X \in \mathbb{C}^{2 n},|\Im X|<\rho\right\}
$$


where $\Im X=\left(\Im X_{1}, \cdots, \Im X_{2 d}\right)$ and $|\cdot|$ is the Euclidean norm in $\mathbb{R}^{2 d}$ for the Hermitean norm in $\mathbb{C}^{2 d}$. So we assume there exist $\rho>0, C>0, \nu \geq 0$, such that $H$ is holomorphic in $\Omega_{\rho}$ and for all $X \in \Omega_{\rho}$, we have

$$
|H(X)| \leq C \mathrm{e}^{\nu|X|} .
$$

Then the $N$-dependent constant $c_{N}$ in (4.9) can be estimated by

$$
c_{N} \leq C^{N+1}(N+1)^{\frac{N+1}{2}}
$$

From this estimate we get an approximation for $U(t) \varphi_{z}$ modulo an exponentially small error (see also [17]).

(iii) There exist $\tau>0, a>0, k>0$ such that for $N=\left\{\frac{a}{\hbar}\right\}$ (the nearest integer to $\left.\frac{a}{\hbar}\right)$, we have

$$
\left.\left.\left\|U(t) \varphi_{z}-\psi_{z, t}^{(N)}\right\| \leq k \mathrm{e}^{-\frac{\tau}{\hbar}}, \forall \hbar \in\right] 0,1\right] .
$$

Now we apply the above estimates and the results already proven [10] concerning the action of metaplectic transformations on Gaussians. Our aim is to study the fidelity

$$
f_{\delta, z}(t)=\left|\left\langle U_{0}(t) \varphi_{z}, U_{\delta}(t) \varphi_{z}\right\rangle\right|^{2}
$$

We shall add the index $\delta$ to keep track of the dependence on the perturbation parameter in the Hamiltonian $H_{\delta}$. $z$ is fixed so we shall omit index $z$.

We use the approximants $\psi_{z, t, \delta}^{(N)}$ and $\psi_{z, t, 0}^{(N)}$ for both terms of the scalar product in (4.14). This yields that to get the result of Theorem $4.2 \bmod \mathcal{O}(\sqrt{\hbar})$ we have to calculate:

$$
\left\langle\hat{T}^{(\hbar=1)}\left(\frac{z_{t}^{0}-z_{t}^{\delta}}{\sqrt{\hbar}}\right) \hat{R}^{1}\left(F_{t}^{0}\right) g, \hat{R}^{1}\left(F_{t}^{\delta}\right) g\right\rangle
$$

But (4.15) is simply of the form

$$
\left.e^{i \beta_{t} / \hbar}\left\langle\hat{T}^{(\hbar=1)}\left(F_{t}^{0}\right)^{-1}\left(\frac{z_{t}^{0}-z_{t}^{\delta}}{\sqrt{\hbar}}\right)\right) g, \hat{R}^{1}\left(\left(F_{t}^{0}\right)^{-1} F_{t}^{\delta}\right) g\right\rangle
$$

where

$$
\beta_{t}:=-\frac{1}{2} \sigma\left(z_{t}^{\delta}, z_{t}^{0}\right) .
$$

Recall that $\sigma(X, Y) \equiv X \cdot J Y$ is the symplectic form in $\mathbb{R}^{2 d}$. 
We are just left with the calculation of the matrix elements of the metaplectic operator between two different coherent states. We have thus established the following important result [10] (here everything is independent of $\hbar$ and we have denoted $\left.g_{X}:=\hat{T}^{(\hbar=1)}(X) g\right)$ :

Lemma 4.5 The matrix elements of $\hat{R}^{1}(F)$ on coherent states $g_{X}$ are given by the following formula:

$$
\begin{gathered}
\left\langle g_{Y+\frac{X}{2}} \mid \hat{R}^{1}(F) g_{Y-\frac{X}{2}}\right\rangle= \\
2^{d}\left(\operatorname{det} V_{t}\right)^{-1 / 2} \exp \left\{\left(K_{F}-\mathbb{1}\right) Y \cdot Y+\frac{i}{2} \sigma\left(X, Y-K_{F} Y-\tilde{K}_{F} Y\right)+\frac{1}{4} J K_{F} J X \cdot X\right\}
\end{gathered}
$$

where $K_{F}:=(\mathbb{1}+F)(\mathbb{1}+F+i J(\mathbb{1}-F))$ and $\tilde{K}_{F}$ is the transpose of $K_{F}$.

Remark 4.6 If $\operatorname{det}(\mathbb{1}+F) \neq 0$, then

$$
K_{F} \equiv\left(\mathbb{1}+i J(\mathbb{1}-F)(\mathbb{1}+F)^{-1}\right)^{-1}
$$

In this case $\hat{R}^{1}(F)$ has a smooth Weyl symbol given by the following formula: (see [10] where we have named this formula the "Mehlig-Wilkinson formula", according to the physics literature [22])

$$
R(F, X)=e^{i \pi \nu}|\operatorname{det}(\mathbb{1}+F)|^{-1 / 2} \exp \left(-i J(\mathbb{1}-F)(\mathbb{1}+F)^{-1} X \cdot X\right)
$$

where $\nu$ is the Maslov index that we have computed exactly.

Moreover we have:

Lemma 4.7 For any symplectic matrix $F$, consider the matrix $V_{F}$ defined by (4.6). We have that $\left|\operatorname{det} V_{F}\right| \geq 1$, and $\left|\operatorname{det} V_{F}\right|=1$ if and only if $F$ is unitary.

Proof of Lemma 4.7; Let

$$
F=\left(\begin{array}{cc}
A & B \\
C & D
\end{array}\right)
$$

be the 4 -block decomposition of the $2 d \times 2 d$ symplectic matrix $F$. We have the following diagonalization property of the Hermitian matrix $i J$ :

$$
i J=U\left(\begin{array}{cc}
-\mathbb{1} & 0 \\
0 & \mathbb{1}
\end{array}\right) U^{*}
$$

where $U$ is the unitary matrix

$$
U=\frac{1}{\sqrt{2}}\left(\begin{array}{cc}
\mathbb{1} & \mathbb{1} \\
i \mathbb{1} & -i \mathbb{1}
\end{array}\right)
$$


Thus we have:

$$
V_{F}=\frac{1}{2} U\left(\left(\begin{array}{ll}
2 & 0 \\
0 & 0
\end{array}\right) U^{*} F U+\left(\begin{array}{ll}
0 & 0 \\
0 & 2
\end{array}\right)\right) U^{*}
$$

and therefore

$$
\begin{gathered}
\operatorname{det} V_{F}=\operatorname{det} \frac{1}{2}\left\{\left(\begin{array}{cc}
A+D+i(B-C) & A-D-i(B+C) \\
0 & 0
\end{array}\right)+\left(\begin{array}{ll}
0 & 0 \\
0 & 2
\end{array}\right)\right\} \\
=\operatorname{det} \frac{1}{2}\left(\begin{array}{cc}
A+D+i(B-C) & A-D-i(B+C) \\
0 & 2
\end{array}\right)=\operatorname{det} \frac{1}{2}(A+D+i(B-C))
\end{gathered}
$$

We conclude that (recall that $\tilde{A}$ is the transpose of the matrix $A$ ):

$$
\left|\operatorname{det} V_{F}\right|^{2}=\operatorname{det} \frac{1}{4}[\tilde{A}+\tilde{D}-i(\tilde{B}-\tilde{C})][A+D+i(B-C)]=\operatorname{det}\left[\mathbb{1}+L^{*} L\right]
$$

with

$$
L=\frac{1}{2}[A-D+i(B+C)]
$$

where we have used the symplecticity of $F$, namely that

$$
\begin{gathered}
\tilde{A} C-\tilde{C} A=\tilde{D} B-\tilde{B} D=0 \\
\tilde{A} D-\tilde{D} A=\mathbb{1}
\end{gathered}
$$

End of Proof of Theorem 4.2: Putting $X=2 Y=\frac{1}{2 \sqrt{\hbar}} F_{0, t}^{-1}\left(z_{0, t}-z_{\delta, t}\right)$ in (4.16), we get (4.5).

Now the estimate (4.7) easily follows from the following:

Lemma 4.8 Let

$$
\gamma_{F}(X)=\frac{1}{4} X \cdot \Gamma_{F} X
$$

Then for any $X \in \mathbb{R}^{2 d}$ we have:

$$
\Re\left(\gamma_{F}(X)\right) \leq-\frac{|X|^{2}}{2\left(1+s_{F}\right)}
$$

where $s_{F} \equiv\|F\|^{2}$ is the largest value of $F \tilde{F}$ ( $\tilde{F}$ being the transpose of the matrix $F)$. 
Proof: let us begin to assume that $\operatorname{det}(\mathbb{1}+F) \neq 0$. Then we have:

$$
K_{F}=(\mathbb{1}+i N)^{-1} \text { where } N=J(\mathbb{1}-F)(\mathbb{1}+F)^{-1} \text { is real symmetric }
$$

so we can compute

$$
\Re\left(K_{F}\right)=\left(\mathbb{1}+N^{2}\right)^{-1}=K_{F} K_{F}^{*} \text { and } \Im\left(K_{F}\right)=-N\left(\mathbb{1}+N^{2}\right)^{-1}
$$

So we get:

$$
\Re \gamma_{F}(X)=\frac{1}{4}\left((\mathbb{1}+J N) K_{F} K_{F}^{*}(\mathbb{1}-N J) X \cdot X-2|X|^{2}\right)
$$

By definition of $K_{F}$, we have:

$$
(\mathbb{1}+J N) K_{F}=2\left((\mathbb{1}+i J) F^{-1}+\mathbb{1}-i J\right)^{-1}:=2 T_{F}
$$

We have, using that $F$ is symplectic

$$
\left(T_{F}^{*}\right)^{-1} T_{F}^{-1}=2\left(\tilde{F}^{-1} F^{-1}+\mathbb{1}\right)
$$

Hence we get:

$$
\begin{gathered}
T_{F} T_{F}^{*}-\frac{\mathbb{1}}{2}=\left(2\left(\tilde{F}^{-1} F^{-1}+\mathbb{1}\right)\right)^{-1}-\frac{\mathbb{1}}{2}=-\frac{\mathbb{1}}{2(\mathbb{1}+\tilde{F} F)} \\
T_{F} T_{F}^{*} X \cdot X-\frac{|X|^{2}}{2}=-\frac{1}{2}(\mathbb{1}+\tilde{F} F)^{-1} X \cdot X \leq-\frac{1}{2\left(1+s_{F}\right)}|X|^{2}
\end{gathered}
$$

and the conclusion follows for $\operatorname{det}(\mathbb{1}+F) \neq 0$, hence for every symplectic matrix $F$ by continuity.

Acknowledgements We thank Jens Bolte for communicating ref. [2] before publication.

\section{References}

[1] Benenti G., Casati G., Veble G., On the Stability of Classically Chaotic Motion under System's Perturbations, Phys. Rev. E, 67, 055202 (R), (2003)

[2] Bolte J., Schwaibold T., Stability of wave packet dynamics under perturbations, Phys. Rev E, (2006) 
[3] Bouzouina A., Robert D., Uniform semiclassical estimates for the propagation of observables, Duke Mathematical Journal 111, 223-252, (2002).

[4] Charbonnel, AM., Localisation et développement asymptotique des éléments du spectre conjoint d'opérateurs pseudodifférentiels qui commutent (Localization and asymptotic development of the joint spectrum of commuting pseudodifferential operators). (French) Integral Equations Oper. Theory 9, 502-536 (1986).

[5] Cerruti N., Tomsovic S., A Uniform Approximation for the Fidelity in Chaotic Systems, J. Phys. A: Math. Gen., 36, 3451-3465, (2003)

[6] Colin de Verdière Y., Bohr-Sommerfeld rules to all orders (To appear in Annales H.P.)

[7] Combescure M., About Quantum Revivals, Quantum Fidelity. A Semiclassical Approach, J. Phys. A (Math. Gen.), 38, 2635-2655, (2005)

[8] Combescure M., The Quantum Fidelity for the time-dependent Singular Quantum Oscillator, to appear in JMP (2006)

[9] Combescure. M., Robert. D., Semiclassical Spreading of Quantum Wavepackets and Applications near Unstable Fixed Points of the Classical Flow, Asymptotic Analysis, 14, 377-404, (1997)

[10] Combescure M., Robert D., Quantum quadratic Hamiltonians revisited, to appear in CUBO Math Journal (2006)

[11] Combescure. M, Ralston. J, Robert. D, A Proof of the Gutzwiller Semiclassical Trace Formula using Coherent Sates Decomposition. Commun. in Math. Phys. 202, 463-480 (1999)

[12] Cucchietti F. M., Dalvit D. A., Paz J. P., Zurek W. H., Decoherence and the Loschmidt Echo, Phys. Rev. Lett. 91, 210403 (2003)

[13] Eckhardt B., Echoes in Classical Dynamical Systems, J. Phys. A: Math. gen., 36, 371-380, (2003)

[14] Emerson J., Weinstein Y. Lloyd S., Cory D., Fidelity Decay as an Indicator of Quantum Chaos, Phys. Rev. Lett., 89, 284102 (2002) 
[15] Fiete G. A., Heller E. J., Semiclassical Theory of Coherence and Decoherence, Phys. Rev. A, 68, 022112, (2003)

[16] Giovannetti V., Llyod S., Maccone L., Quantum Limits to Dynamical Evolution, Phys. Rev. A, 67, 052109 , (2003)

[17] Hagedorn G., Joye A., Semiclassical dynamics with exponentially small error estimates, Comm. Math. Phys., 207, 449-465, (1999)

[18] Helffer B., Robert D., Puits de potentiel généralisés et asymptotique semiclassique, Annales de l'IHP (section Physique théorique), 41, No 3, 291-331, (1984)

[19] Jacquod P., Adagideli I., Beenakker C. W., Decay of the Loschmidt Echo for Quantum States with sub-Planck scale Structures, Phys. Rev. Lett., 89, 154103, (2002)

[20] Leichtle C., Averbukh I., Schleich W.,Multilevel quantum beats: An analytical approach, Phys. Rev. A, 54, 5299-5312, (1996)

[21] Littlejohn. R The semiclassical evolution of wave packets, Physics Reports, 138, Nos 4\& 5, (1986)

[22] Mehlig B., Wilkinson M., Semiclassical trace formulae using coherent states Ann. Phys. (Leipz),10, 541, (2001)

[23] Parker.J., Stroud. J. Coherence and Decay of Rydberg Wave Packets Phys.Rev. Lett. 56, p.716-719 (1986)

[24] Peres A., Stability of Quantum motion in Chaotic and Regular Systems, Phys. Rev. A 30, 1610-1615, (1984)

[25] Prosen T., On General Relation Between Quantum Ergodicity and Fidelity of Quantum Dynamics, Phys. Rev. E 65, (2002)

[26] Prosen T., Seligman T. H., Znidaric M., Stability of Quantum Coherence and Correlation Decay, Phys. Rev. A, 67, 042112 , (2003)

[27] Prosen T., Seligman T. H., Znidaric M., Theory of Quantum Loschmidt Echoes, Prog. Theo. Phys. Supp. 150 , 200-228 , (2003) 
[28] Prosen T., Znidaric M., Stability of Quantum Motion and Correlation Decay, J. Phys. A , Math. Gen, 35, 1455-1481, (2002)

[29] Robert. D., Autour de l'approximation semi-classique, Birkhäuser, Progress in Mathematics, 68, (1987)

[30] Robert. D., Remarks on time dependent Schrödinger equation, bound states and coherent states, in : Optimal Control and Partial Differential Equations, IOS Press, 188-197, (2001)

[31] Robert. D., Propagation of coherent states in quantum mechanics and applications

Proceedings of the CIMPA summer school in Lanzhou. To appear (2006)

[32] Robinett R. W. Quantum wavepackets revivals, Phys. Rep. 392, 1-119, (2004)

[33] Silvestrov P. G., Tworzydlo J., Beenakker C. W., Hypersensitivity to Perturbations of Quantum-Chaotic Wavepacket Dynamics, Phys. Rev. Lett. 67, 025204- (R), (2003)

[34] Vanicek J., Heller E. J., Semiclassical Evaluation of Fidelity, Phys. Rev. E, $68,056208,(2003)$

[35] Weinstein Y., Lloyd S., Tsallis C., Border between Regular and Chaotic Quantum Dynamics, Phys. Rev. Lett., 89, 214101, (2002)

[36] Znidaric M., Prosen T., Fidelity and Purity Decay in Weakly Coupled Composite Systems, J. Phys. A, Math. Gen., 36, 2463-2481, (2003) 